\title{
Extending the Limits of Place and Temporal Pitch Perception in Cochlear Implant Users
}

\author{
Olivier Macherey ${ }^{1,2}$, John M. Deeks ${ }^{1}$, and Robert P. Carlyon ${ }^{1}$ \\ ${ }^{1}$ Medical Research Council, Cognition and Brain Sciences Unit, 15 Chaucer Road, Cambridge, CB2 7EF, UK \\ ${ }^{2}$ Laboratoire de Mécanique et d'Acoustique, 31 Chemin Joseph Aiguier, Marseille, 13009, France
}

Received: 23 June 2010; Accepted: 26 October 2010; Online publication: 30 November 2010

\begin{abstract}
A series of experiments investigated the effects of asymmetric current waveforms on the perception of place and temporal pitch cues. The asymmetric waveforms were trains of pseudomonophasic (PS) pulses consisting of a short, high-amplitude phase followed by a longer (and lower amplitude) opposite-polarity phase. When such pulses were presented in a narrow bipolar ("BP+1") mode and with the first phase anodic relative to the most apical electrode (so-called PSA pulses), pitch was lower than when the first phase was anodic re the more basal electrode. For a pulse rate of 12 pulses per second (pps), pitch was also lower than with standard symmetric biphasic pulses in either monopolar or bipolar mode. This suggests that PSA pulses can extend the range of place-pitch percepts available to cochlear implant listeners by focusing the spread of excitation in a more apical region than common stimulation techniques. Temporal pitch was studied by requiring subjects to pitchrank single-channel pulse trains with rates ranging from 105 to 1,156 pps; this task was repeated at several intra-cochlear stimulation sites and using both symmetric and pseudomonophasic pulses. For PSA pulses presented to apical electrodes, the upper limit of temporal pitch was significantly higher than that for all the other conditions, averaging 713 pps. Measures of discriminability obtained using the method of constant stimuli indicated that this pitch percept was probably weak. However, a multidimensional scaling study showed that the percept associated with a rate change, even at high rates, was orthogonal to that of a
\end{abstract}

Correspondence to: Olivier Macherey - Medical Research Council, Cognition and Brain Sciences Unit 15 Chaucer Road, Cambridge, CB2 7EF, UK. Telephone: +44-1223-355294; fax: +44-1223-359062; email: olivier.macherey@mrc-cbu.cam.ac.uk place change and therefore reflected a genuine change in the temporal pattern of neural activity.

Keywords: temporal pitch, place pitch, electrical stimulation

\section{INTRODUCTION}

Pitch cues can be conveyed to cochlear implant (CI) listeners along two perceptually independent dimensions corresponding to the locus of excitation along the cochlea (referred to as "place pitch") and to the repetition rate of the electrical waveform ("temporal pitch"; Tong et al. 1983; McKay et al. 2000; Kong and Carlyon 2010). Both of these cues suffer from limitations. First, the range of place pitches is limited by the fact that electrodes are usually not inserted all the way into the apex of the cochlea. Second, most studies of temporal pitch perception reveal an "upper limit" of about 300-500 pulses per second (pps), beyond which changes in repetition rate do not produce an increase in pitch (Townshend et al. 1987; Zeng 2002; Baumann and Nobbe 2004; Carlyon et al. 2010a).

In a recent publication, Middlebrooks and Snyder (2010) reported the phase-locked activity of inferior colliculus (IC) neurons in response to electrical stimulation of the auditory nerve of anaesthetized cats. They showed that neurons could phase lock to higher rates when neural information originated from the apex of the cochlea compared to when it originated from other cochlear sites. Specifically, IC units with characteristic frequencies (CFs) below $1,500 \mathrm{~Hz}$ had the highest limiting rates, with some of 
them being able to phase lock to the stimulus up to $600 \mathrm{pps}$, the highest rate tested. In contrast, units with characteristic frequencies higher than $1,500 \mathrm{~Hz}$ showed lower limiting rates, usually below 400 pps. Middlebrooks and Snyder (2010) further suggested that their results reflected the existence of a specific pathway originating at the apex of the cochlea and specialized for high temporal acuity. If this result applies to human CI listeners, we would expect temporal pitch perception, which relies on phase locking, to be better when stimulating apical rather than basal electrodes. However, previous studies comparing the upper limits of temporal pitch at different cochlear locations did not show any superiority of apical stimulation (Zeng 2002; Baumann and Nobbe 2004; Carlyon et al. 2010b). One possible reason for this is that the electrode currents did not stimulate the apex selectively enough and that the temporal information coming from apical fibres was blurred by more basal fibres projecting to IC neurons with a lower limiting rate. The present study examines this hypothesis using different stimuli and methods than previously used.

Our stimuli are based on the observations that short phases are more effective (i.e. need less charge to evoke the same loudness) than longer phases (Shannon 1985; Moon et al. 1993; Macherey et al. 2006) and that anodic (positive) phases are more effective than cathodic (negative) ones (Macherey et al. 2006, 2008, 2010; van Wieringen et al. 2008; Undurraga et al. 2010). Figure 1 schematizes some expected spatial excitation patterns in response to different pulse shapes. For symmetric biphasic pulses presented to a monopolar channel (Fig. 1 a), we expect a broad excitation pattern centred on the active electrode (here the most apical). In the case of bipolar stimulation (Fig. $1 \mathrm{~b}-\mathrm{d}$ ), each intra-cochlear electrode is stimulated with reference to another nearby intra-cochlear electrode. This can be viewed as stimulating simultaneously both electrodes with opposite-polarity pulses. When using symmetric pulses (Fig. $1 \mathrm{~b}$ ), the pulse will be anodic relative to the more apical electrode during the first phase and anodic re the more basal electrode during the second phase, thereby creating similar amounts of excitation in the vicinity of both electrodes. However, by using pseudomonophasic pulses with a short, high-amplitude phase anodic relative to the most apical electrode ("PSA" in Fig. $1 \mathrm{c}$ ), we expect nerve fibres proximal to the most apical electrode to be more effectively excited than fibres proximal to the other electrode (because the "effective" anodic short phase is presented on the more apical electrode). The opposite pattern should be obtained if the polarity is reversed (Fig. $1 \mathrm{~d}$ ). Some recent masking and pitch data collected in a wide "BP +9 " bipolar configuration corroborated this hypothesis

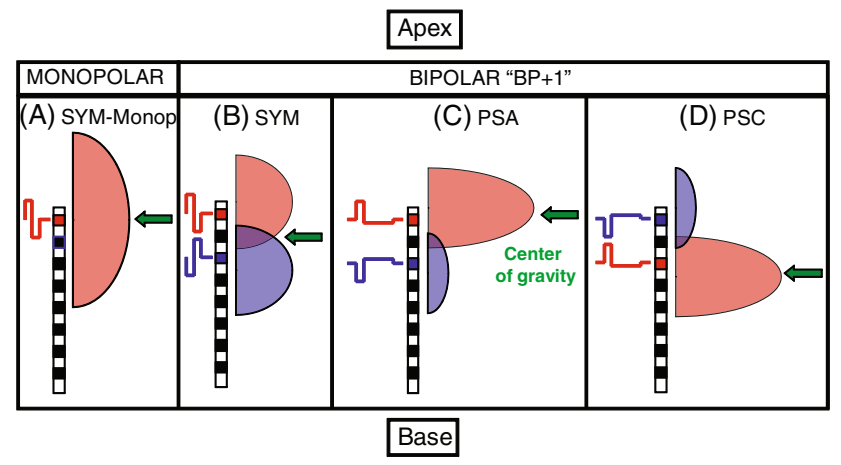

FIG. 1. Schematic representation of expected spatial excitation patterns produced by four different electrical pulse shapes at the apex of the cochlea.

(Macherey et al. 2010). The aims of the present study were to extend these results to narrower bipolar configurations $(\mathrm{BP}+1)$ and to investigate their impact on pitch perception.

The following series of experiments is based on the hypothesis that PSA pulses can focus the spatial spread of excitation at the apex of the cochlea (Fig. 1 c). We show that, at least at low pulse rates, these stimuli elicit a lower place-pitch percept than symmetric pulses presented in monopolar or in bipolar mode and that they allow the subject to perceive increases in temporal pitch up to higher rates than for other intra-cochlear stimulation sites and/or pulse shapes. We also show that the "upper limit" for temporal pitch correlated negatively, across waveform shape and site of stimulation, with the current level needed to reach a comfortable loudness. This and other results suggest that, for the subjects tested here, selective stimulation of the cochlear apex may improve temporal pitch perception at high rates via a more effective electrode-neuron interfaceperhaps resulting from better neural survival-rather than by activating a central pathway dedicated to fine temporal processing.

\section{EXPERIMENT 1: PRELIMINARY STUDY}

\section{Rationale and methods}

Rationale. This experiment was designed (1) to test the general idea that bipolar pseudomonophasic pulses presented at the apex can produce lower place-pitch percepts than bipolar symmetric pulses and (2) to test whether such stimuli can increase the upper limit of temporal pitch. We first compare the place pitch produced by different waveform shapes and modes of stimulation and do this for pulse trains at three different rates. We then measure the temporal pitch produced by a wide range of pulse rates and compare the results obtained with different waveform shapes and stimulation modes. 
Subjects and stimuli. Seven users (S1-S7) of the Advanced Bionics CII/HiRes 90k device took part. Their biographical data are indicated in Table 1 . Testing was approved by the Cambridge Local Research Ethics Committee. Subjects were paid for participating. Stimuli were $400-\mathrm{ms}$ unmodulated pulse trains presented on a single channel of the implant in bipolar "BP +1 " mode (with an inter-electrode distance of $2.2 \mathrm{~mm}$ ) or in monopolar mode (with the case electrode as the return contact). In bipolar mode, we define polarity in terms of the polarity of the more apical electrode. The phase duration was always $97 \mu$ s except for pseudomonophasic pulses for which the duration of the second phase was increased by a factor of 4 and its amplitude reduced by the same factor to maintain charge balancing. Stimuli were presented through the APEX experimental software platform (Laneau et al. 2005) which acts as an interface for the BEDCS software provided by Advanced Bionics. There were three sets of bipolar stimuli differing in their pulse shape and intracochlear site: pseudomonophasic anodic at the apex ("PSA-Apex" on electrodes 1 and 3), symmetric biphasic anodic-first at the apex ("SYM-Apex", also on electrodes 1 and 3) and symmetric biphasic anodic-first in the middle ("SYM-Middle" on electrodes 7 and 9). For each set, there were eight different stimuli with rates ranging from 105 to 859 pps. These eight stimuli were logarithmically spaced with a difference between consecutive rates of approximately $35 \%$. There were also three identical sets of monopolar stimuli. The active electrode was electrode 1 for the "PSA-Apex-Monop" and "SYMApex-Monop" conditions and electrode 8 for the "SYM-Middle-Monop" condition.

Loudness balancing. The stimuli were first loudness balanced using a procedure similar to that used by Macherey and Carlyon (2010). For each pair of stimuli that had to be balanced, at least two adjustments were performed. In one adjustment, one of the two stimuli (the standard) was fixed at most comfortable loudness (MCL) and the other stimulus (the signal) was adjusted by the subject. In the second adjustment, standard and signal were swapped to correct for possible biases due to order effects. For some subjects, this bias was strong and it could happen that stimulus B was adjusted at a lower level than stimulus A during the first adjustment but that stimulus A was then adjusted at a lower level than stimulus B during the second adjustment. In this case, the procedure was repeated two or four more times. Standard and signal were presented one after the other with a gap of $500 \mathrm{~ms}$. The subjects could adjust the level of the signal by pressing six different buttons corresponding to steps of 16,32 and $48 \mu \mathrm{A}$ in both directions and were encouraged to "bracket" their matches. Each time they pressed a button, the two sounds were played again, and this was repeated until they judged the sounds as having the same loudness. The balanced level of the signal was then calculated by averaging the level differences (in decibels) between standard and signal obtained in the different adjustments and subtracting this value from the standard's initial level. On some occasions (especially at low pulse rates), the averaged level difference revealed by the adjustment procedure was smaller than the minimum step size.

The adjustments were performed in two steps. First, the lowest-rate stimuli (105 pps) of the three stimulus sets were equated in loudness. The SYM-Apex stimulus was fixed at MCL, and both the PSA-Apex and the SYM-Middle were loudness balanced to it. Then, for each individual set, the 258-pps stimulus was balanced to the 105 pps, the 479 pps to the 258 pps and the $859 \mathrm{pps}$ to the $479 \mathrm{pps}$. Levels for intermediate rate stimuli were obtained by logarithmic interpolation.

The decrease in MCLs as a function of rate was $-1.4 \mathrm{~dB}$ per decade on average (pooled across conditions and subjects). This value is comparable to that found in previous studies for similar ranges of rates (e.g. Kreft et al. 2004).

Place pitch in bipolar mode. The place-pitch difference between SYM-Apex and PSA-Apex was assessed in a twointerval, two-alternative forced-choice task. In each trial,

\begin{tabular}{|c|c|c|c|c|c|}
\hline \multicolumn{6}{|c|}{ TABLE 1} \\
\hline \multicolumn{6}{|c|}{ Subjects' biographical details } \\
\hline & Age & Aetiology & Duration of deafness & Implant use & Implant type \\
\hline$\overline{S 1}$ & 50 & Progressive & Unknown (onset at 33 years) & 1 year & HR90k \\
\hline S2 & 78 & Unknown & Unknown & 1 year & HR90k \\
\hline S3 & 78 & Progressive & Unknown (onset at 56 years) & 1 year & HR90k \\
\hline S4 & 65 & Progressive & $>12$ years & 1.5 years & HR90k \\
\hline S5 & 74 & Unknown sudden & $<2$ years & 7 years & CII \\
\hline S6 & 55 & Meningitis & 1 year & 7 years & $\mathrm{CII}$ \\
\hline S7 & 62 & Unknown progressive & 34 years & 6 years & HR90k \\
\hline S8 & 19 & Mumps & 6 months & 1 year & HR90k \\
\hline S9 & 49 & Idiopathic & 2.5 years & 6 months & HR90k \\
\hline
\end{tabular}


subjects listened to a SYM-Apex followed by a PSA-Apex stimulus (or vice versa) presented at the same rate. They had to indicate which stimulus had the higher pitch by pressing one of two virtual buttons displayed on a computer screen. The comparison was performed at three different rates $(105,258$ and $644 \mathrm{pps})$ which were mixed in blocks of 60 trials (20 repetitions per comparison). Three blocks were performed for each subject (leading to 60 trials per comparison) except S7 who only did two. No feedback was provided.

Temporal pitch in bipolar mode. The upper limit of temporal pitch was determined for the three different stimulus conditions using the mid-point comparison procedure, which has been described in detail elsewhere (Long et al. 2005; Macherey and Carlyon 2010). Briefly, it consists of making pitch comparisons between pairs of sounds. The choice of sounds to be presented on a given trial is driven by the results of previous trials in such a way that the whole set of stimuli can be pitch-ranked in a minimum of comparisons. By repeating the procedure several times, a mean and a standard error of the rank are obtained for each stimulus. The three conditions were run in separate blocks presented in alternation and in a randomized order (which differed across subjects). Depending on time available within the session, between ten and 12 blocks per condition were collected. No feedback was provided.

Compared to common methods used to measure temporal pitch discrimination (e.g. rate difference limens, cf. Baumann and Nobbe 2004), this procedure has two advantages and one disadvantage. First, because pulse rate varies a lot from trial to trial, it helps the subjects focus on the pitch dimension. For example, a subject may have to compare in one trial two stimuli which differ a lot in pitch (e.g. 105 vs. $479 \mathrm{pps})$ followed, in another trial, by two stimuli which are much more similar (e.g. 479 and 644 pps). Second, because no feedback is provided, we do not assume that pitch increases monotonically over the whole range of rates. We have previously reported that the pitch sensation can sometimes go down with increases in pulse rate, and we will show that this also happened in the present experiment (Carlyon et al. 2010a). Giving feedback in this context would most likely confuse the subjects in his/her following judgments. Finally, a disadvantage of this procedure is that any error on a given trial-for example, produced by a lapse in attention-will affect the rank order of the whole set of stimuli. To correct for these errors, obvious outliers were excluded from the data as follows: We computed the standard deviations of the ranks of each stimulus and removed individual ranks which were higher or lower than the mean rank plus or minus 2.6 times the standard deviation, respectively (corresponding to values lying outside the $99 \%$ confidence interval of the normal distribution). Because any error on one comparison will affect the ranking of the whole set of stimuli, an entire run was removed each time an outlier was detected. On average, less than one run was removed per condition and per subject.

Temporal pitch in monopolar mode. In a subsequent session, the exact same procedure was repeated with a subset of subjects (S1-S4 and S6) using monopolar stimuli. The step sizes used for the loudness balancing procedure were reduced to 4,8 and $12 \mu \mathrm{A}$. Only the temporal pitch part of the experiment was performed.

\section{Results and discussion}

Place pitch in bipolar mode. Figure 2 shows the percentage of trials where PSA-Apex was judged lower in pitch than SYM-Apex for the three different rates. The bars showing the percentage scores are shown relative to chance level $(50 \%)$. When the bar is in the upper part of the graph (as is mostly the case), it means PSA-Apex was lower than SYM-Apex whereas a bar in the lower part means it was higher. Mean and 95\% confidence intervals are illustrated on the right of the panel and indicate that overall PSA-Apex elicited a significantly lower pitch than SYM-Apex at the three rates tested. A one-way repeated-measure analysis of variance (ANOVA) using the Huynh-Feldt correction also showed a significant effect of rate $(F(1.6,9.7)=9.69, p=0.021)$. The pitch difference between PSA-Apex and SYM-Apex was larger at $258 \mathrm{pps}$ than at 105 pps $(p=0.004)$ and 644 pps $(p=0.031)$.

One possible explanation for this rate effect relates to interfering temporal pitch cues. At 105 pps, temporal pitch cues are presumably more salient than at higher rates (cf. Moore and Carlyon 2005). When estimating the pitch of a given stimulus, subjects have to weigh the two dimensions of place and rate. It is possible that the weight applied to the temporal pitch dimension is relatively larger at $105 \mathrm{pps}$ than at

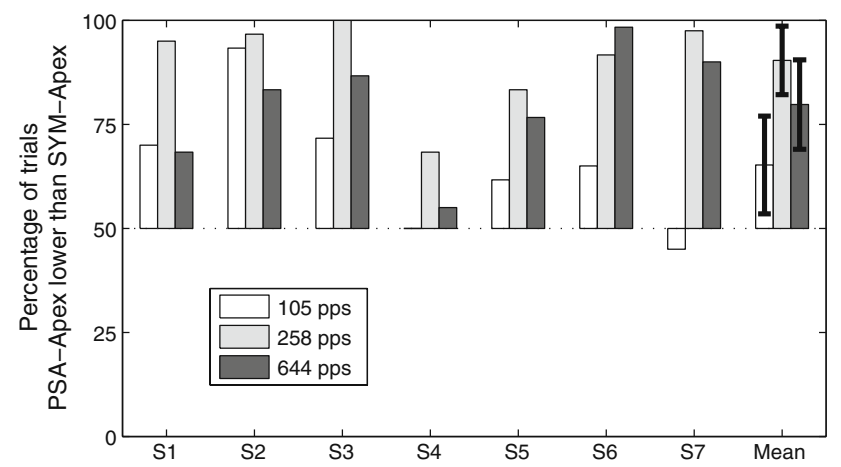

FIG. 2. Results of the place-pitch part of experiment 1 (bipolar mode). The bars show the percentage of times PSA-Apex was judged lower than SYM-Apex when presented at the same rate. The error bars show the $95 \%$ confidence intervals. 
258 pps, thereby obscuring any weaker place-pitch differences. This argument, however, cannot explain the smaller place-pitch difference observed at 644 than at 258 pps because temporal pitch cues should become weaker with increases in pulse rate. Place-pitch cues will be specifically and more thoroughly investigated in experiment 3, and we will propose several possible interpretations for this effect of rate.

Temporal pitch in bipolar mode. The panels $A-G$ of Figure 3 illustrate the temporal pitch results for individual subjects (filled squares for PSA-Apex, open circles for SYM-Apex and open triangles for SYM-Middle). Although the three functions showing the mean ranks and standard errors are shown on the same panels, it is important to recall that the three conditions were performed in different blocks of trials. Here, we are interested in finding, for each function, the rate above which the sensation of pitch ceases to increase (i.e. the rate above which the rank function flattens off). It can be seen that, for subjects S1-S6, the PSA-Apex rank function continues to increase up to at least 644 pps. The PSA-Apex function of S6 shows a peculiar trend with a first increase in pitch, followed by a plateau between 191 and 344 pps and by another increase for higher rates. The results for the other two conditions are more variable across subjects. For S7, the three functions reach their maximum below 300 pps after which the pitch sensation asymptotes or even decreases with increases in rate. Such non-monotonic trends have been observed previously by Carlyon et al. (2010a).

The data were analysed in two different ways detailed in the "Appendix". Both analyses yielded similar results. In the first analysis, we determined the upper limit of temporal pitch by fitting broken-stick functions (comprising one portion increasing linearly as a function of the logarithm of the rate followed by a second portion with zero slope) to each data set. The upper limit was defined as the rate at which the two portions of the broken-stick fit intercept. In the second analysis, we determined the slope of increase of the pitch rank for rates above 300 pps (i.e. for rates above the commonly reported upper limit of temporal pitch). An advantage of this latter analysis over the first one was the possibility to compare statistically (by calculating a $t$ value) the slopes of the different conditions within each subject. The values of upper limit and slope for the different subjects and conditions are given in Table 2 .

Panel $\mathrm{H}$ shows the mean upper limit as determined by fitting broken-stick functions to the ranking data. A one-way repeated-measure ANOVA performed on the $\log$ of the upper limits failed to show a significant effect of condition $(F(1.4,8.4)=3.43, p=0.091)$. The
(A) $\mathrm{S} 1$
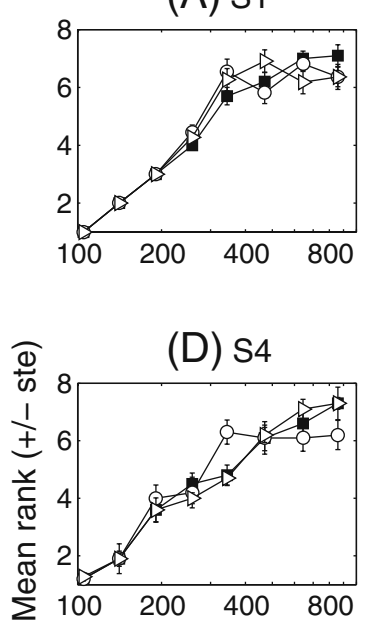

(G) $\mathrm{S7}$

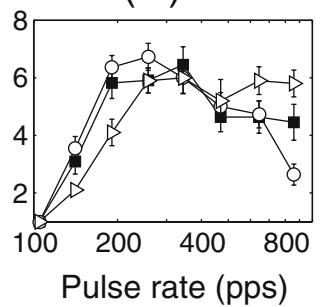

(B) $\mathrm{s} 2$

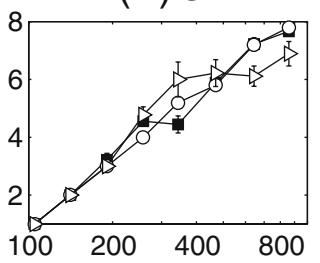

(E) $\mathrm{S} 5$

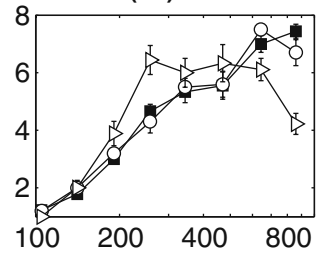

(C) $\mathrm{s} 3$

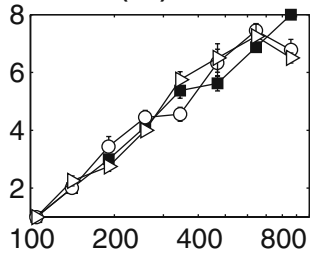

(F) 56

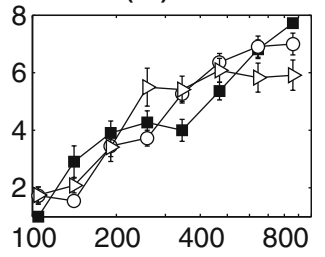

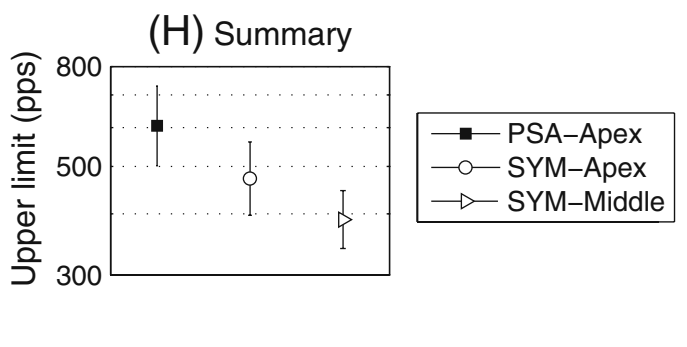

FIG. 3. Results of the temporal pitch part of experiment 1 (bipolar mode). A-G Individual results. $\mathbf{H}$ Mean and standard error of the upper limit of temporal pitch (pooled across subjects). 
TABLE 2

Upper limits of temporal pitch, slopes of increase of the temporal pitch-rank functions above 300 pps and current levels needed for MCLs obtained in experiments 1 and 2

\begin{tabular}{|c|c|c|c|c|c|c|c|c|c|}
\hline Experiment & Analysis & Condition & S1 & S2 & $S 3$ & $S 4$ & S5 & S6 & $S 7$ \\
\hline \multirow{6}{*}{$\begin{array}{l}\text { Experiment } 1 \\
\text { (bipolar) }\end{array}$} & \multirow[t]{3}{*}{ Upper limits (pps) } & PSA-Apex & 563 & 776 & 859 & 602 & 732 & 859 & 209 \\
\hline & & SYM-Apex & 363 & 790 & 620 & 355 & 644 & 595 & 216 \\
\hline & & SYM-Middle & 399 & 388 & 515 & 707 & 258 & 361 & 258 \\
\hline & \multirow[t]{3}{*}{ Slopes } & PSA-Apex & 0.5 & 0.89 & 0.87 & 0.56 & 0.64 & 0.87 & -0.33 \\
\hline & & SYM-Apex & 0.04 & 0.92 & 0.56 & 0 & 0.46 & 0.55 & -0.62 \\
\hline & & SYM-Middle & -0.07 & 0.2 & 0.3 & 0.75 & -0.38 & 0.07 & -0.08 \\
\hline \multirow{6}{*}{$\begin{array}{r}\text { Experiment } 1 \\
\text { (monopolar) }\end{array}$} & \multirow[t]{3}{*}{ Upper limits (pps) } & PSA-Apex & 344 & 547 & 859 & 517 & - & 859 & - \\
\hline & & SYM-Apex & 484 & 859 & 859 & 310 & - & 859 & - \\
\hline & & SYM-Middle & 543 & 436 & 469 & 615 & - & 297 & - \\
\hline & \multirow[t]{3}{*}{ Slopes } & PSA-Apex & -0.22 & 0.58 & 1 & 0.47 & - & 0.82 & - \\
\hline & & SYM-Apex & 0.24 & 0.9 & 1 & 0.32 & - & 0.82 & - \\
\hline & & SYM-Middle & 0.52 & 0.33 & 0.29 & 0.69 & - & 0.02 & - \\
\hline \multirow{12}{*}{$\begin{array}{l}\text { Experiment } 2 \\
\text { (bipolar) }\end{array}$} & \multirow[t]{4}{*}{ Upper limits (pps) } & PSA-Apex & 684 & 832 & 798 & 1,146 & 1,001 & 859 & - \\
\hline & & PSC-Apex & 430 & 619 & 350 & 366 & 801 & 801 & - \\
\hline & & PSA-Middle & 447 & 461 & 543 & 699 & 329 & 442 & - \\
\hline & & SYM-Middle & 469 & 389 & 396 & 613 & 344 & 258 & - \\
\hline & \multirow[t]{4}{*}{ Slopes } & PSA-Apex & 0.67 & 0.66 & 0.68 & 0.84 & 0.81 & 0.89 & - \\
\hline & & PSC-Apex & 0.23 & 0.49 & -0.13 & 0.2 & 0.61 & 0.75 & - \\
\hline & & PSA-Middle & 0.13 & 0.41 & 0.54 & 0.72 & 0.29 & 0.32 & - \\
\hline & & SYM-Middle & 0.34 & 0.21 & -0.25 & 0.68 & -0.65 & 0.08 & - \\
\hline & \multirow[t]{4}{*}{ MCLs (dB re $1 \mathrm{~mA})$} & PSA-Apex & -3.88 & -4.79 & -7.29 & -6.38 & -4.55 & -6.23 & - \\
\hline & & PSC-Apex & -3.05 & -3.45 & -6.38 & -5.55 & -3.45 & -5.55 & - \\
\hline & & PSA-Middle & -2.86 & -0.95 & -6.97 & -6.67 & -3.45 & -1.27 & - \\
\hline & & SYM-Middle & -3.88 & -1.43 & -6.9 & -6.97 & -3.05 & -2.57 & - \\
\hline
\end{tabular}

The slopes of three apical stimuli of experiment 1 (PSA-Apex, SYM-Apex and SYM-Apex-Monop) were compared by calculating $t$ values (cf. "Appendix"). PSA-Apex showed significantly greater slopes than SYM-Apex for S1, S3, S4 and S6 $(p<0.001)$ and no difference for S2 and S5. PSA-Apex also showed greater slopes than SYM-Apex-Monop for S1 and S4 $(p<0.05)$, no difference for S2 and S6 and a significantly smaller slope for S3 ( $p<0.01)$. Finally, the slopes obtained with SYM-Apex-Monop were significantly greater than those obtained with SYM-Apex for S3, S4 and S6 $(p<0.01)$ but showed no difference for S1 and S2

same statistical test performed on the slope values indicated a main effect of condition $(F(1.5,9.0)=4.75$, $p=0.047)$ with PSA-Apex showing significantly greater slopes than both SYM-Apex $(p=0.021)$ and SYMMiddle $(p=0.022)$. It is worth noting that, for at least four subjects, the upper limit in the PSA-Apex condition was probably underestimated because of ceiling effects, which, more generally, may have obscured differences between conditions. An extended range of rates will be investigated in experiment 2 .

Temporal pitch in monopolar mode. As a control and in order to check that the higher upper limit of temporal pitch obtained with PSA-Apex, as measured by the steeper slopes at high rates in that condition, was not due to the particular temporal distribution of charge delivered by this pulse shape, we also performed the temporal pitch part of the experiment with a subset of subjects using monopolar stimuli. For the five subjects tested ( $\mathrm{S} 1-\mathrm{S} 4$ and $\mathrm{S} 6$ ), the slope of increase above 300 pps for monopolar PSA-Apex was either the same or was lower than that for monopolar
SYM-Apex (cf. Table 2). Furthermore, a paired-sample $t$ test showed the difference in slope between PSA-Apex and SYM-Apex to be significantly greater in bipolar than in monopolar mode $(p=0.019)$. This demonstrates that the improvement at high rates obtained with PSA-Apex in bipolar mode is not due to the particular shape of the pulse.

The differences in slope between SYM-Apex and SYM-Middle were consistent with those obtained in bipolar mode. S1 and S4 were better in the middle (S4 was already better in the middle with bipolar and S1 showed no difference). The other three subjects were better at the apex (as in bipolar mode) for which they performed at ceiling. Comparing the slopes of PSA-Apex bipolar to those of PSA-Apex-Monop and SYM-Apex-Monop showed an inconsistent pattern of results across subjects. Although these comparisons were not the main focus of this study (the conditions were performed on different days and may have been obscured by ceiling effects), they are interesting in several ways and will be further discussed in experiment 3 . 


\section{EXPERIMENT 2: TEMPORAL PITCH CUES}

Rationale and methods

Experiment 2 aimed to extend the temporal pitch results obtained in experiment 1. First, we wanted to re-examine the upper limit of temporal pitch with bipolar PSA-Apex pulses using an extended range of rates to avoid ceiling effects. Second, we wanted to investigate alternative reasons for the superiority of PSAApex. Computational and animal model data have shown that pseudomonophasic pulses in bipolar mode can produce a more place-specific excitation pattern than symmetric ones (Frijns et al. 1996; Bonham et al. 2003). It is then possible that the improvement observed in experiment 1 was due to this increased specificity rather than just stimulating apically. If this is the case, we should obtain similarly good performance using the same pulse shape at a different location.

We repeated the same procedure as in experiment 1 with a shifted range of rates (seven rates from 191 to 1,159 pps) with subjects S1-S6. This time, four bipolar conditions were compared: PSA-Apex, PSC-Apex (i.e. the same as PSA-Apex but with reversed polarity), PSA-Middle and SYM-Middle. The same pairs of electrodes were tested as in experiment 1 ( 1 and 3 for the apex conditions; 7 and 9 for the middle conditions) and the same stimulus parameters (phase duration and stimulus duration) were used. If the improvement is due to the pulse shape itself, then a similar upper limit should be obtained for PSA-Apex, PSC-Apex and PSA-Middle. However, if PSA-Apex is found to be better than the other conditions, this would indicate that there is something specific about using this particular pulse shape at the apex.

As these data were collected on a different day than those of experiment 1, we first checked that the levels used in experiment 1 for PSA-Apex and SYM-Middle still evoked the same loudness sensation. For the subjects for whom it was the case (i.e. all subjects except S1 and S3), the first six stimuli of both sets were kept at the same level (these rates were identical to the ones used in experiment 1). Only the 1,146-pps stimulus was balanced to the 859 pps for both conditions. For the other two subjects, the levels were re-measured from scratch. The levels for the other two conditions (PSC-Apex and PSA-Middle) were obtained in a similar way as in experiment 1: The 191-pps stimuli were loudness balanced across conditons with reference to SYM-Middle. Then, for each set of stimuli, the 344 pps was balanced to the $191 \mathrm{pps}$, the $644 \mathrm{pps}$ to the $344 \mathrm{pps}$ and the 1,146 pps to the 644 pps. The levels for intermediate rates were obtained by logarithmic interpolation.

\section{Results and discussion}

The panels A-F of Figure 4 illustrate the individual results for three of the four conditions (filled squares
(A) S1
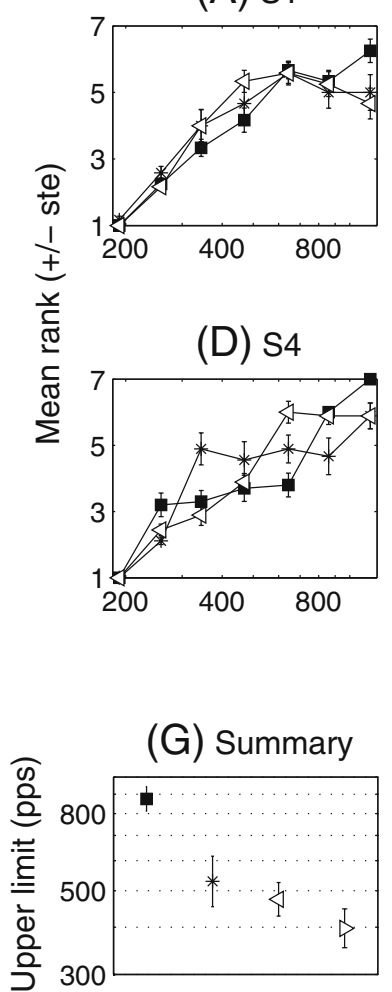

(B) $\mathrm{s} 2$

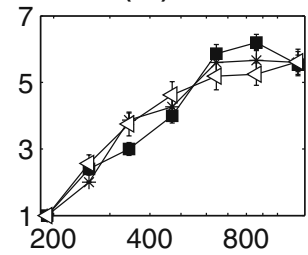

(E) S5

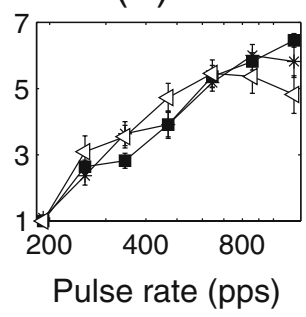

(C) $\mathrm{s} 3$

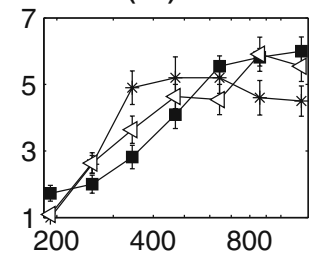

(F) S6

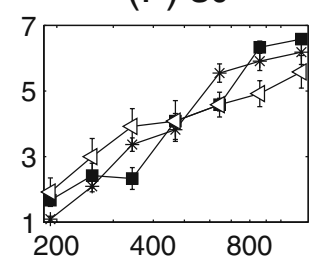

$$
\begin{aligned}
& \square \text { PSA-Apex } \\
& * \text { PSC-Apex } \\
& \neg \text { PSA-Middle } \\
& \longrightarrow \text { SYM-Middle }
\end{aligned}
$$

FIG. 4. Results of experiment 2. A-F Individual results. G Mean and standard error of the upper limit of temporal pitch (pooled across subjects). 
for PSA-Apex, asterisks for PSC-Apex and open triangles for PSA-Middle). The results for SYM-Middle were very similar to those obtained in experiment 1 (saturated at approximately the same rates or were non-monotonic) and are not shown here. Again, for the six subjects, the rank function of PSA-Apex increases up to very high rates (more than $644 \mathrm{pps}$ ) while the other conditions show less consistent results across subjects (cf. Table 2). It is, however, worth noting that for any given subject, it was usually the case that pitch increased up to high rates for one condition other than PSA-Apex, although which condition this was differed across subjects. In other words, good temporal pitch perception at high rates is possible with a range of cochlear sites and waveform shapes but only occurred consistently for PSA stimuli at the apex.

The same analyses as in experiment 1 were performed on the data (cf. "Appendix"). Panel G shows the mean and standard errors of the upper limits as determined by fitting broken-stick functions. A repeated-measures ANOVA performed on the log of the upper limit data revealed a main effect of condition $(F(1.6,8.0)=7.28, p=0.019)$. The upper limit for PSA-Apex was significantly higher than that for PSC-Apex $(p=0.029)$, PSA-Middle $(p=0.002)$ and SYM-Middle $(p=0.001)$. We also compared the upper limits of PSA-Apex and SYM-Middle taking into account the results of S7 who only did experiment 1 . A paired-sample $t$ test revealed that the mean upper limit was significantly higher $(p=0.011)$ for PSA-Apex (713 pps) than for SYM-Middle (374 pps). The same statistical analysis performed on the slopes yielded very similar results both qualitatively and quantitatively. There was a main effect of condition $(F(2.3,11.4)=6.13$, $p=0.014)$ with PSA-Apex showing greater slopes than PSC-Apex $(p=0.012)$, PSA-Middle $(p=0.009)$ and SYM-Middle $(p=0.004)$.

Although the higher upper limit of temporal pitch, obtained with the PSA stimuli at the apex, is consistent with better central processing of apical stimulation (Middlebrooks and Snyder 2010), we wanted to test whether the PSA stimuli could have increased the upper limit of temporal pitch for another reason. Specifically, it has been suggested that there is a more efficient electrode-neuron interface at the apex than at the base of the cochlea, perhaps resulting from better neural survival (Fayad and Linthicum 2006; Bierer 2007). As a more efficient electrode-neuron interface would be likely to require less current to activate AN fibres, we investigated whether the current level needed for comfortable loudness at 191 pps correlated, across conditions, with the upper limit of temporal pitch. Within-subject correlations were combined using an analysis of covariance approach, as described by Bland and Altman (1995). The MCLs (in decibels re $1 \mathrm{~mA}$ ) were indeed found to be negatively correlated with the log of the upper limits $(r=-0.75, d f=17, p<0.001)$. A significant correlation was also obtained between the MCLs and the slopes $(r=-0.57, d f=17, p=0.011)$. An additional one-way ANOVA analysis was performed on the upper limits of the four conditions with MCL as a covariate. In this case, the effect of condition was not significant anymore $(p=0.39)$. This relation between the upper limit of temporal pitch and the current level needed to reach a particular loudness strongly suggests a peripheral component to the differences in performance observed between the different conditions. Note that, although we did not measure detection thresholds for the different conditions, we would not expect them to correlate with the upper limits because polarity sensitivity does not hold at threshold (Macherey et al. 2006). We would therefore expect PSA-Apex and PSC-Apex to show very similar thresholds.

Finally, because our experimental procedure does not use level roving, there is a possibility that residual loudness cues may have influenced the pitch judgments. However, in an additional set of measures (results not shown), we repeated the mid-point comparison procedure for PSA-Apex stimuli using a limited range of rates. The particularity of this measure was that the different rate stimuli had the same current level per pulse. It has been shown that loudness can have an effect on pitch and that it is more often the case that louder stimuli are associated with higher pitches (Carlyon et al. 2010b). If the good performance obtained with PSA-Apex was solely due to residual loudness cues, we would have expected most subjects to perform better when the level is kept constant (because loudness cues should be even more salient). However, for the five subjects tested, this was not the case and performance was either the same or worse when the current level was kept the same than when the stimuli were loudness balanced. This observation argues against a major influence of loudness on the temporal pitch results of experiment 2.

\section{EXPERIMENT III: PLACE-PITCH CUES}

\section{Rationale and methods}

Place-pitch comparisons between electric stimuli. In experiment 1, we found that bipolar asymmetric pulses presented at the apex could create lower place-pitch percepts than symmetric biphasic ones. We also found that there was a significant effect of rate on the magnitude of the pitch difference. Because the upper limit of temporal pitch differed across conditions, this effect may have been at least partly caused by interfering temporal pitch cues. Here we investigate place-pitch differences at two different 
rates for which the influence of temporal cues should be limited: a very low rate of 12 pps and a fairly high rate of 1,031 pps. At 12 pps, subjects can hear individual pulses, and this rate is below the lower limit of temporal pitch measured in normal-hearing listeners, thereby minimizing any influences of phase locking on the pitch percept (Krumbholz et al. 2000). At 1,031 pps, temporal pitch cues should be weak as this rate is higher than the averaged upper limit measured in experiment 2. Furthermore, the placepitch comparisons of experiment 1 were limited to bipolar pulses while contemporary CI strategies almost all use monopolar stimulation. Therefore, we also extended our place-pitch comparison of PSA-Apex to a regular monopolar symmetric biphasic pulse train presented on the most apical electrode of the implant. This allowed us to determine whether we could get even more apical stimulation than with monopolar coupling. Finally, to check that these place-pitch differences were not restricted to the apex, we performed some of the comparisons at another intra-cochlear site.

Place-pitch comparisons were performed using a two-interval forced-choice task. For each rate, there were two blocks of trials. In block 1 , four different comparisons were performed: PSA-Apex vs. PSC-Apex, PSA-Apex vs. SYM-Apex-Monop and the same two comparisons in the middle of the array (electrodes 7 and 9 for bipolar and electrode 7 for monopolar for all subjects except $\mathrm{S} 8$ and $\mathrm{S} 9$ for whom the electrodes tested were 8 and 10). In block 2, there were three comparisons: PSA-Apex vs. SYM-Apex, PSA-Apex vs. SYM-Apex-Monop (same as in block 1) and SYM-Apex vs. SYM-Apex-Monop. The stimuli were loudness balanced by first measuring MCLs for PSA-Apex and PSA-Middle. Then, all apical stimuli were balanced with reference to PSA-Apex, and all "middle" stimuli were balanced with reference to PSA-Middle. Eight subjects (S1-S6, S8 and S9) performed the comparison at 12 pps; six (S1-S6) did so at 1,031 pps.

Place-pitch comparisons between acoustic and electric stimuli. S8 and S9 are unusual subjects in that they were unilaterally deaf prior to implantation and have normal hearing in their non-implanted ear. They are part of an ongoing study designed to investigate the usefulness of CIs for tinnitus alleviation (Dauman et al. 2009) and are referred to as "C1" and "C2" in a recent study on pitch matching between electric and acoustic stimuli (Carlyon et al. 2010c). To quantify the size of the place shift obtained with PSA-Apex compared to SYM-Apex-Monop, we asked these two subjects to directly compare the pitches evoked by these two stimuli to the pitches evoked by several acoustic stimuli presented to their contralateral ear. These two subjects had already performed pitch comparisons between their two ears and showed consistent results for several electrodes, including the most apical one.

These direct comparisons were performed using 12-pps acoustic and electric pulse trains, also to limit the contribution of temporal pitch to the pitch sensation. The acoustic stimuli were bandpass filtered harmonic complexes generated by summing a large number of components $(\mathrm{F} 0=6 \mathrm{~Hz})$ in alternating sine and cosine phase; this phase relationship produced a pulse rate of 12 pps, which is double the F0. The amplitudes of the nine components closest to the filter centre frequency $(\mathrm{CF})$ were equal, and component amplitude decreased at a rate of $48 \mathrm{~dB} /$ octave outside that passband. A large number of stimuli, with (centre) frequencies ranging from 250 to $9944 \mathrm{~Hz}$ in steps of $2 \%$, were generated in advance and stored as waveform files on a laptop computer. The duration of each waveform file was $500 \mathrm{~ms}$ including 10 -ms linear ramps. The stimuli were presented at $70 \mathrm{~dB}$ SPL via an Edirol UA-25 external sound card and one earpiece of a Sennheiser HD650 headset. Prior to the pitch comparisons, the levels of the two electric sounds were adjusted to match the loudness of the acoustic sounds.

Each block of trials consisted of two interleaved adaptive procedures (Jesteadt 1980), which converged on the $29 \%$ and $71 \%$ points of the psychometric function, respectively, that is, points where the acoustic stimulus was judged higher than the electric stimulus on $29 \%$ and $71 \%$ of trials. The point of subjective equality (PSE) was estimated from the geometric means of these two values. The initial (centre) frequencies of the acoustic stimuli used for the two tracks differed by two octaves and were the same for both electric standards (471 and $1,884 \mathrm{~Hz})$.

\section{Results and discussion}

\section{At 12 pps}

Apical stimulation. The left-hand panel of Figure 5A shows the results obtained for each individual subject when 12-pps pulse trains were presented to the most apical electrodes of the array. For each subject, the white, light grey and dark grey bars show the percentage of trials on which PSA-Apex was judged lower in pitch than PSC-Apex, SYM-Apex and SYM-Apex-Monop, respectively. The bars on the right show the mean and 95\% confidence intervals and demonstrate that overall PSA-Apex had a significantly lower pitch than each of the others. Assuming that at $12 \mathrm{pps}$ temporal pitch cues do not affect the pitch comparisons, this suggests that the centre of gravity of the excitation pattern produced by PSA-Apex is more apical than that produced by all the other pulse shapes. This observation corroborates the initial hypothesis of this study. The pattern of results for individual subjects indicates that this was true for all 


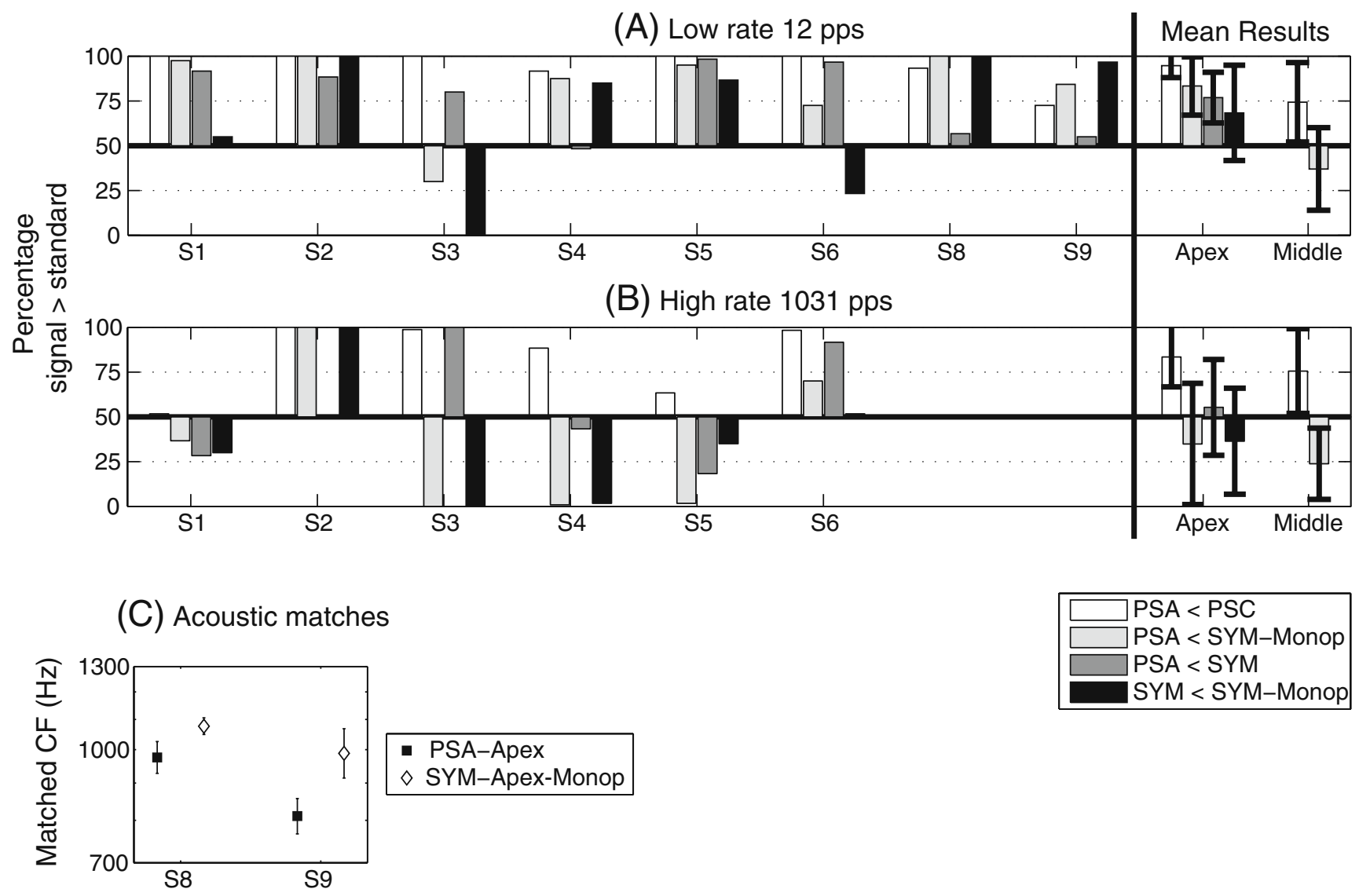

FIG. 5. Results of experiment 3. A Place-pitch comparisons at 12 pps. B Place-pitch comparisons at 1,031 pps. C Acoustic-to-electric pitch comparisons for the two unilateral subjects (S8 and S9).

subjects except S3 for whom SYM-Apex-Monop was the lowest and S4, S8 and S9 for whom SYM-Apex had the same pitch as PSA-Apex. Furthermore, there was no consistent pitch difference between bipolar and monopolar symmetric biphasic pulses, as shown by the black bars.

It is now important to investigate the relation between these place-pitch comparisons and the upper limits of temporal pitch collected in the first two experiments. Two observations can be made:

1. PSA-Apex evokes a lower place pitch than both PSC-Apex and SYM-Apex and also has a higher upper limit of temporal pitch, as measured in experiments 1 and 2.

2. The only subject (S3) for whom SYM-Apex-Monop was lower in pitch than PSA-Apex is the only subject for whom temporal pitch perception was better (significantly higher slope of increase) with SYM-Apex-Monop than with PSA-Apex in experiment 1 (cf. Table 2).

Five of the subjects (S1-S4 and S6) who performed the bipolar and monopolar temporal pitch conditions of experiment 1 also performed the present experiment. The three apical stimuli (PSA-Apex, SYM-Apex and SYM-Apex-Monop) were ranked from the best to the worst in terms of upper limit of temporal pitch assuming that one condition was better than another if their slopes were significantly different from each other ( $p<0.05$ using the $t$ calculation described in the "Appendix"). Significant differences are indicated in Table 2. The same stimuli were also ranked from the lowest to the highest in terms of place pitch assuming that one stimulus was different from another if it was judged more than $70 \%$ of the time lower or higher in pitch. These two rank parameters were found to be modestly but significantly correlated $(r=0.63, d f=9$, $p=0.038$ ). This finding suggests that more apical stimulation may allow the subjects to perceive increases in temporal pitch up to higher rates.

Middle of the array. In the middle of the electrode array, individual subjects' data were less consistent than at the apex and only the mean and 95\% confidence intervals are shown (far right of the panel). Overall, PSA-Middle was also significantly lower than PSC-Middle but was not significantly different from SYM-Middle-Monop (the results varied a lot from one subject to another). This observation will be discussed later in the paper. 
At 1,031 pps. The left-hand panel of Figure 5B shows the results obtained for each individual subject when 1,031-pps pulse trains were presented to the most apical electrodes of the array. The results were much more variable across subjects than was the case at 12 pps, and although PSA was judged to have a significantly lower pitch than PSC, it was not judged to be consistently lower than the symmetric biphasic stimuli. One possible explanation for this is that a higher upper limit of temporal pitch for the PSA stimulus counteracted a lower place pitch. We are currently investigating this and other possible explanations which are detailed in the general discussion of the present paper. Mean data for stimulation of the middle part of the electrode array are shown in the far right of Figure 5B. PSA was found to be significantly lower in pitch than PSC but significantly higher than SYM-Middle-Monop. The reason for this unexpected trend is at present unclear.

Direct comparisons with acoustic stimuli. Figure 5C shows the results of the direct acoustic-to-electric pitch comparisons. It illustrates for both subjects (S8 and S9) the acoustic centre frequencies equivalent to PSA-Apex (filled squares) and to SYM-Apex-Monop (open diamonds) at 12 pps. We already know from Figure 5A that PSA-Apex sounds lower than SYMApex-Monop for these two subjects, but these direct comparisons provided additional information on the magnitude of this pitch difference.

The PSE decreased from 1,077 to $976 \mathrm{~Hz}$ for S8 and from 988 to $811 \mathrm{~Hz}$ for S9 when using PSA-Apex instead of SYM-Apex-Monop. Paired-sample $t$ tests (one-tailed) revealed that these differences were significant ( $p=0.040$ for S8 and $p=0.017$ for S9).

\section{EXPERIMENT 4: INDEPENDENCE OF PLACE AND TEMPORAL PITCH CUES}

\section{Rationale and methods}

Experiment 4 tested the hypothesis that the increase in the upper limit of temporal pitch obtained with PSA-Apex was caused by place-of-excitation cues. This alternative explanation was entertained because, as suggested in a previous study, polarity sensitivity may depend on current level (Macherey et al. 2010). Because the current level of high-rate stimuli needs to be reduced to match the loudness of lower-rate stimuli, it is possible that the increase in temporal pitch observed with PSA-Apex above 300 pps was due to this level reduction, which could reduce the polarity sensitivity and therefore cause a basal shift in place of excitation. In other words, it is possible that the increase in pitch as a function of rate reflected a place rather than a temporal pitch change.
Experiment 4 was performed to test for this possible effect.

Five subjects (S1-S4 and S6) participated in a multidimensional scaling (MDS) study. There were 12 different stimuli differing in their stimulation site (PSA-Apex and PSC-Apex, both on electrodes 1 and 3, and PSA-Middle on electrode 5 and 7) and in their pulse rate (four rates spaced by $35 \%$ ). The rates were chosen to be the four highest rates for which each subject could perceive an increase in pitch in the PSAApex condition. These rates differed across subjects (258-644 pps for S1-S4; 344-859 pps for S6). The 12 stimuli were first loudness balanced. This was done by first balancing the lowest-rate stimuli of the three stimulation sites together (with PSA-Apex as the reference). Then, for each condition, the three highest rates were balanced to the lowest rate. In the main part of the experiment, stimuli were presented in pairs, and the subjects were asked to estimate the amount of dissimilarity between them by pointing on a 10-cm unmarked line. All possible pairs were randomly presented in the two possible orders, leading to a total of 120 estimations per block. Dissimilarity estimates were converted to numbers by linear subdivisions of the $10-\mathrm{cm}$ line. These numbers were then grouped together to form a dissimilarity matrix. Between five and nine matrices were collected with each subject during one or two testing sessions. The first matrix of each session counted as training and was not taken into account in the analysis.

The several dissimilarity matrices were then analysed for each subject separately using a nonmetric, multidimensional scaling algorithm (ALSCAL; Young et al. 1978). Because each analysis involved dissimilarity data collected in one subject, the data were treated as unconditional (MacCallum 1977). We expected two possible outcomes for the MDS solution.

1. Place changes and rate changes do not reflect independent perceptual dimensions. This would imply that place-of-excitation co-varies with rate (due to, e.g. a change in current level).

2. Place changes and rate changes correspond to independent and orthogonal dimensions. This would imply that the improved upper limit obtained with PSA-Apex is genuine and reflects a change in the temporal pattern of neural activity.

\section{Results and discussion}

The results of the MDS analysis are illustrated in Figure 6. Each panel shows the graphical representation of the solution for individual subjects S1, S2, S3 and S6. A three-dimensional solution modelled the 

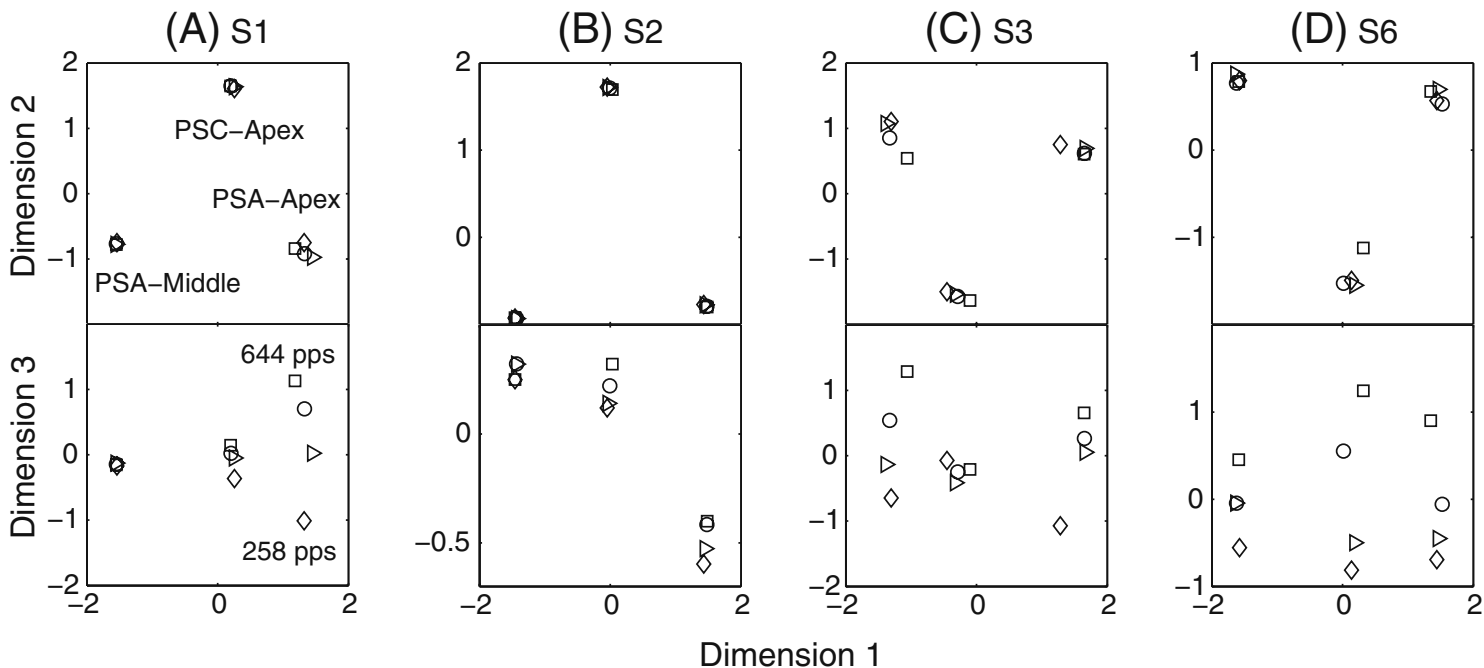

FIG. 6. A-D Results of the multidimensional scaling study of experiment 4 . Dimensions 1 and 2 correspond to the type of stimulation; dimension 3 corresponds to the pulse rate. For S2, the dissimilarity judgments were well modelled by a two-dimension solution only showing the three types of stimulation. However, the three-dimension solution is shown for comparison with other subjects.

responses with Kruskal's stress (formula 1) values of $0.1,0.01,0.13$ and 0.15 for S1, S2, S3 and S6, respectively. The proportion of variance of the data accounted for by the three-dimension solutions was in every case more than $84 \%$. The data of S 4 were very variable across matrices and showed a much higher stress value for the three-dimension solution $(0.23)$. In a further analysis, we found that his dissimilarity ratings across the whole session were negatively correlated with trial number $(r=-0.44, p<0.0001)$. In other words, he found all pairs of sounds to be very different at the beginning of the session and the same pairs to be very similar at the end of the session. This can be viewed as a type of contraction bias sometimes observed in magnitude estimation studies (Poulton 1979). This non-sensory bias reflects a situation where a subject tends to give ratings close to the middle of the range once he is used to the stimuli. Given this lack of consistency across repetitions, the data of S4 are not shown here.

The top panels of Figure 6 show the first two dimensions of the solution, which appear to correspond to the place of stimulation. For the four subjects, the solution shows the exact same trend: There are three spatially separated groups of four stimuli corresponding to the three sites or types of stimulation (PSA-Middle, PSC-Apex and PSA-Apex from left to right). Because the ALSCAL solution is invariant to rotation of the coordinate system, the first two axes were rotated so that the three groups of stimuli would correspond to the expected change in place pitch along dimension 1 (high to low from left to right). The observation that the three stimulus types do not scale on a single dimension means that the subjects did not consistently rate the dissimilarity as being larger when comparing PSA-Apex and PSA-
Middle than when comparing PSA-Apex and PSCApex or PSC-Apex and PSA-Middle, despite the fact that the place-pitch difference was presumably larger. This may relate to two possible things. First, MDS data are often better modelled using one more dimension than is present physically. This finding has been reported previously (so-called horse-shoe effect) and can be attributed to a non-linear relationship between the perceptual differences between pairs of stimuli and the numbers that subjects assign to these differences (Kendall 1971; McKay and Carlyon 1999; McKay et al. 2005). In the present experiment, this might simply be due to the fact that subjects had difficulties ranking the three stimulus types because they were very distinct from each other. It is therefore strongly possible that the three stimulus types only differ along one perceptual dimension (i.e. place pitch) but that the MDS algorithm needs two dimensions to correctly fit the data. Second, it cannot be excluded that these two dimensions correspond to two perceptually distinct dimensions, one being the place of stimulation and the other corresponding to the pulse shape (e.g. PSA vs. PSC). We should stress, however, that the horse-shoe effects are ubiquitous in MDS studies, even when there is no obvious possibility for an additional perceptual dimension.

If increases in rate produced changes in place of excitation, we would expect the high rates of PSAApex to get closer to those of PSC-Apex along dimension 1 and/or 2. Contrary to this hypothesis, the bottom panels of Figure 6 show that dimension 3, which reflects differences in pulse rate, is orthogonal to that of a change in place (dimensions 1 and 2). For the four subjects, the four rates of the PSA-Apex condition were correctly ordered from the lowest to the highest. This was, however, not the case for the 

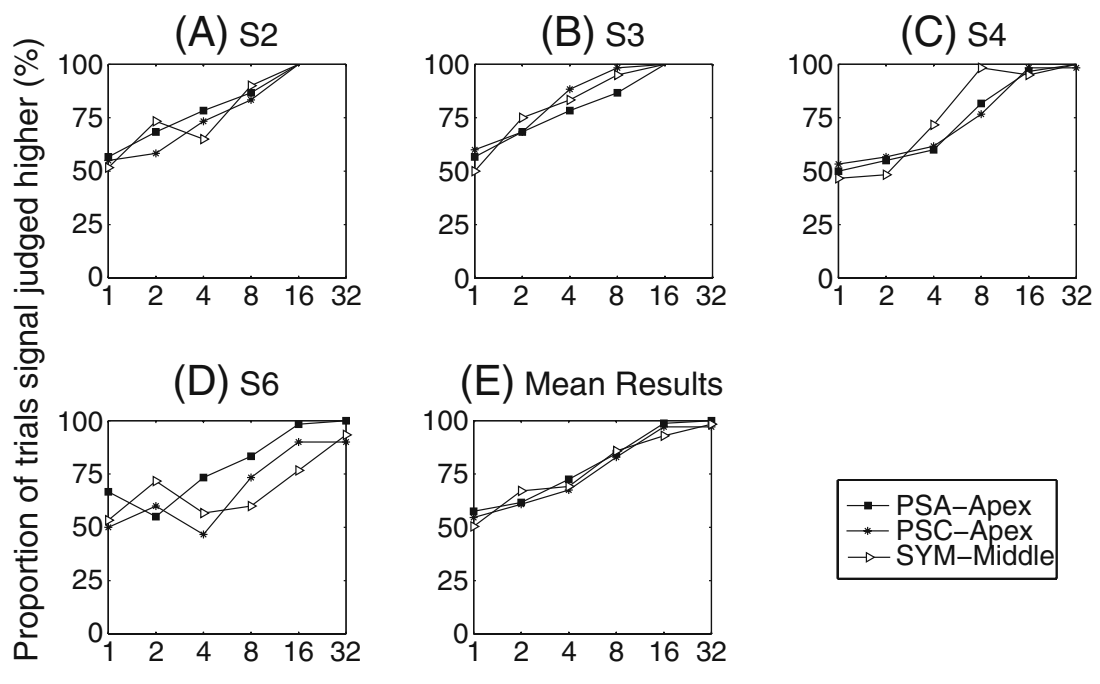

Rate difference (\%)

FIG. 7. A-E Psychometric functions obtained in experiment 5. Proportion of time a higher-rate signal was judged higher in pitch than the 105-pps standard, as a function of the rate difference between standard and signal. This was performed for three different pulses: PSA-Apex, PSC-Apex and SYM-Middle.

other two conditions. It is interesting to note that, for S1 and S3, the rates of the PSC-Apex condition were perceptually more similar to each other than were the rates of the PSA-Apex condition. For S2 and S6, however, the PSC-Apex rates were as well ordered as the PSA-Apex ones. These observations are consistent with the temporal pitch results of experiment 2 where S1 and S3 performed poorly with PSC-Apex whereas S2 and S6 performed almost as well with PSC-Apex as with PSA-Apex.

\section{EXPERIMENT 5: STRENGTH OF THE TEMPORAL PITCH PERCEPT}

\section{Rationale and methods}

Although the mid-point comparison allows us to capture the upper limits of temporal pitch in different conditions, it does not provide information on the strength of the percept and on how small a difference the subjects can perceive.

In experiment $5 \mathrm{a}$, we measured rate difference limens (DLs) at three different baseline rates (105, 344 and 644 pps) for PSA-Apex. Five subjects took part in this sub-experiment ( $\mathrm{S} 1-\mathrm{S} 4$ and $\mathrm{S} 6)$. The three baseline rates were first loudness-balanced together. For each condition, a signal with a rate $35 \%$ higher was then balanced to each baseline. If the MCLs of standard and signal were identical, all stimuli were set at the same level. If they were not, the levels were interpolated. The procedure was a two-interval forced-choice, two-down, one-up, adaptive task (Levitt 1971). The change from increasing to decreasing rate or vice versa was called a turn point, and the procedure ended after ten turn points. The initial rate difference was $35 \%$, the same difference as used in the mid-point comparison procedure. The step size of the rate change was $8 \%$ and switched to about $2 \%$ after three turn points. The DL value was calculated as the mean of the last six turn points. Between three and five repetitions were collected at each rate. Because we knew that for this range of rates pitch increased monotonically, feedback was provided.

In experiment $5 \mathrm{~b}$, we investigated whether the higher upper limit of temporal pitch obtained with PSA-Apex pulses would generalize to a more accurate temporal pitch perception at low rates (105 pps). Four subjects participated ( $\mathrm{S} 2-\mathrm{S} 4$ and $\mathrm{S} 6$ ). We used the method of constant stimuli to measure psychometric functions at a 105-pps baseline rate in three different conditions: PSA-Apex, PSC-Apex and SYM-Middle (electrodes 7 and 9). The standard was always 105 pps and was compared to six other stimuli with higher rates than the standard $(1 \%, 2 \%, 4 \%, 8 \%, 16 \%$ or $32 \%$ higher). Feedback was provided and the three conditions were run in separate blocks of 120 trials (20 repetitions of the six comparisons). Three blocks were performed for each condition and each subject (total of 60 comparisons per trial). The order of presentation of the blocks was randomized across subjects.

Prior to performing these pitch comparisons, the 105-pps stimuli of the three conditions were loudness balanced together. The highest-rate signal (32\% higher) of each condition was then loudness balanced to its corresponding standard. The adjusted levels showed that, within this very small range of low rates, MCL was relatively constant. The levels of all stimuli within each condition were therefore kept the same. 
Results and discussion

Rate difference limens. All subjects had rate DLs lower than $5 \%$ at 105 pps, and these could not be accurately measured as the minimum rate difference between standard and signal was set to 2\%. At 344 and $644 \mathrm{pps,}$ however, only S1 and S6 could perform the task. Their averaged DLs across repetitions were 24 and $14 \%$ for S1 and 18 and $16 \%$ for S6, respectively, for 344 and 644 pps baseline rates. The procedure did not converge with the other three subjects, suggesting that their DLs were larger than $35 \%$. We further used the method of constant stimuli (without feedback) and measured the percentage of trials where they could detect a $35 \%$ rate difference at 344 and 644 pps. At least 60 trials were performed at each rate. At $344 \mathrm{pps}$, mean percent correct ranged from $69 \%$ to $77 \%$ whereas it was between 51 and $68 \%$ at 644 pps.

This rather poor performance measured at 344 and 644 pps (DLs equal or higher than 35\%) in three of the subjects suggests that, despite a measurable increase in the upper limit of temporal pitch observed in experiments 1 and 2, it is likely that the temporal pitch percept is still very weak at such high rates. It also seems that the mid-point comparison procedure used in experiments 1 and 2 allowed us to reveal weak but consistent pitch changes.

Psychometric functions at $\mathbf{1 0 5}$ pps. Figure 7 shows the proportion of trials where subjects judged the signal as higher than the standard. For any given subject, performance varied slightly across the different conditions. However, the mean data show that there was no trend of one condition being superior to the others (cf. Fig. 7E). This result indicates that the higher upper limit obtained with PSA-Apex may not translate to better pitch discrimination performance at low rates.

\section{GENERAL DISCUSSION}

Summary of results

1. Bipolar pseudomonophasic pulses presented at the apex of the cochlea elicit lower place-pitch percepts when the short, high-amplitude phase is anodic re the more apical electrode (PSA) than when it is anodic re the more basal electrode. This finding was true for the two stimulation rates tested (12 and 1,031 pps).

2. At $12 \mathrm{pps}$, the place pitch of PSA is also lower than that produced by monopolar or bipolar symmetric biphasic pulses.

3. At 1,031 pps, place-pitch comparisons between PSA and monopolar or bipolar symmetric pulses produced more variable and less consistent results.
4. PSA pulses at the apex also allow the subjects to perceive increases in temporal pitch up to higher rates than with other pulse shapes and/or stimulation sites.

5. There was a negative correlation between the upper limit of temporal pitch and the current level needed to reach most comfortable loudness. This finding suggests that our results are mainly determined by a peripheral factor like, e.g. neural survival.

6. A multidimensional scaling study demonstrated that a change in rate induced a perceptual change orthogonal to that produced by a change in intracochlear stimulation site.

7. Additional pitch measures using the method of constant stimuli suggested that the temporal pitch percept at high rates remains weak with PSA pulses at the apex.

\section{Implications for cochlear implants}

Place pitch. Extending the range of place pitches towards the apex may effectively add another (potentially important) channel of information to implant users. It may also be useful to patients with a partial electrode insertion for whom stimulation is restricted to the basal part of the cochlea. For the two subjects with normal hearing in the contralateral ear, the difference in pitch between bipolar stimulation of the apex with the PSA stimulus and monopolar stimulation of the most apical electrode with a symmetric pulse was $10 \%$ for S8 and $20 \%$ for S9. Those two subjects also took part in another study, in which they adjusted acoustic stimuli to match the pitches produced by monopolar stimulation of several individual electrodes (Carlyon et al. 2010c). That study showed that the matched pitches closely corresponded to the predictions of Greenwood's (1990) frequency-to-place formula. From this formula, we can conclude that the change in pitch produced by the PSA stimulus corresponds to a place shift of between 0.55 and $1.1 \mathrm{~mm}$. In a recent study, Saoji and Litvak (2010) tested another method designed to produce lower place pitches than standard monopolar stimulation of the most apical electrode. Their "phantom electrode" stimuli produced place shifts that were similar in size to the ones reported here.

It is at present unclear why PSA-Apex did not produce a consistently lower pitch than SYM-Apex and SYM-Apex-Monop at 1,031 pps. There may be at least three possible explanations:

1. We found that apical stimulation could convey temporal pitch cues up to higher rates than other stimuli. It is therefore possible that, even at 1,031 pps, PSA-Apex still conveys a higher temporal pitch code than the other stimuli and that 
this cue counteracts a lower place pitch for this stimulus.

2. We have previously failed to find any effect of stimulus polarity at threshold level and suggested that there might be an effect of current level on polarity sensitivity, i.e. that polarity sensitivity may only appear above a certain level (Macherey et al. 2010). To maintain the same loudness as that evoked by a 12-pps stimulus, the current level used at 1,031 pps needs to be reduced. It is possible that, at least for some subjects, this lower current level is below the level at which polarity starts to matter. Note, however, that polarity sensitivity can still be measured in monopolar mode (via loudness differences between PSA and PSC) where current level is much lower than that used in bipolar mode, even at 12 pps (cf. Macherey et al. 2006). Furthermore, it is worth noting that PSA still evoked a consistently lower pitch than PSC at 1,031 pps.

3. A potentially important difference between low and high stimulation rates is the effect of neural adaptation. At high rates, the amplitude of the compound response of the AN shows a marked decrease during the first pulses of the train (Rubinstein et al. 1999). Matsuoka et al. (2000) found that cats' fibres had different degrees of adaptation to anodic and cathodic pseudomonophasic pulses, showing more adaptation to anodic stimuli. If the same applies to our CI users, this would make the response of fibres close to the anodic electrode decrease more rapidly during the train than fibres close to the cathodic electrode, thereby shifting the centre of gravity of the excitation to a more basal site shortly after the onset of the stimulus. We may in this case expect the pitch of a pseudomonophasic pulse train to change over the course of the stimulus. Although they were not explicitly asked, it is worth noting that none of our subjects reported the pitch of PSA or of PSC to change during presentation.

Explanations (2) and (3) would also imply that, as pulse rate is increased, the place of excitation should shift (either because polarity sensitivity decreases due to the current level difference or because of neural adaptation). In both cases, we would therefore expect the site of excitation of PSA and PSC to get closer to each other with increases in rate. The results of the MDS study, which showed two distinct place pitches for PSA and PSC (even for S1 who showed very different place-pitch results at 12 and 1,031 pps), argue against these two explanations and would favour (1).

It is also, at present, unclear why PSA did not produce a lower pitch than SYM-Monop in the middle of the array. This could be due to differences in local neural survival near the two electrodes of the bipolar channel. If, for some subjects, survival is better near the more basal electrode (number 9) than near the more apical electrode (7), PSA may fail to selectively recruit nerve fibres near 7 and produce significant excitation of fibres near 9, thereby eliciting a higher place pitch than monopolar stimulation of electrode 7. Alternatively, this may be due to differences in the current spread produced by monopolar stimulation of apical and middle sites.

Temporal pitch. Consistent with the finding of Middlebrooks and Snyder (2010), our results suggest that selectively stimulating the apex leads to a better transmission of phase-locking cues. This result may appear, at first, counter-intuitive because apical fibres naturally code the lower-frequency part of the spectrum. However, as argued by Middlebrooks and Snyder (2010), auditory nerve fibres with relatively high CFs do not phase lock to their best frequency so it may not be beneficial to have a central pathway preserving fine-structure cues in this case. In contrast, apical auditory nerve fibres do phase lock to their CF, and the cochlear nucleus and inferior colliculus may have evolved to preserve these fine-structure cues. Interestingly, Middlebrooks and Snyder (2010) found that only a small portion $(13-14 \%)$ of the sampled IC units was able to follow a 600-pps pulse train and that, even in those cases, phase locking was weaker than at lower rates. Our data showing large DLs (and weak percept) at high rates are consistent with these results and with the idea that there is a very small portion of units conveying the temporal code at high rates.

We do not know whether the upper limits of temporal pitch that we measured were below or above the CFs of the fibres recruited by PSA-Apex pulses. It is worth noting that the two unilateral subjects S8 and S9 matched the pitch of the PSA-Apex stimulus to acoustic pulse trains with centre frequencies of 976 and $811 \mathrm{~Hz}$, respectively. These matched frequencies are in the ball-park of the upper limits of temporal pitch measured in experiment 2. It could be that, for some subjects, we are getting close to a correspondence between place and rate of stimulation. It has been proposed that such a place-rate match may be necessary for accurate pitch perception in normal hearing (Oxenham et al. 2004), and it would be interesting to investigate any relationships between these two variables in CI users.

In a previous study, Baumann and Nobbe (2004) compared rate discrimination at two different intracochlear sites of Med-El users. Although Med-El implants usually have deep electrode insertions, they did not find any advantage of stimulating the apical region. There may be several reasons for this. First, their most apical electrode was electrode 3 which may not have been apical enough. Second, they used monopolar stimulation. It is therefore possible that 
the portion of apical nerve fibres recruited by their stimulus was relatively small compared to the total population of fibres, due to a lack of spatial selectivity. Last, it has been shown that deep insertions can sometimes have deleterious effects on place-pitch perception. Finley et al. (2008) showed that deep insertions are more likely to cause apical contacts to be in the scala vestibuli which may induce cross-turn stimulation. In another study, Gani et al. (2007) found that two CI subjects with deep insertions also showed pitch confusions between their most apical electrodes.

Our results may be interpreted in two different ways. First, it is possible that they reflect a peripheral factor, namely the efficiency of the electrode-neuron interface. Second, it is also possible that they reflect the existence of a central mechanism able to follow temporal fine-structure cues up to high rates when neural information originates at the apex of the cochlea. The finding that the level of stimulation needed to reach a comfortable percept correlated negatively with the upper limit of temporal pitch may be compatible with both of these explanations. The relatively low MCLs obtained with PSA-Apex may indeed reflect the existence of a better electrodeneuron interface at the apex due to, e.g. a higher neural density and/or a closer positioning of the electrode to the spiral ganglion cells. But they may also be due to a better phase locking at the level of the IC as it could be that regular firing in the IC does sound louder.

Several observations would, however, argue for a peripheral explanation:

1. Some subjects performed almost as well in the middle of the array.

2. The different subjects probably had different electrode insertion depths so it is likely that PSAApex did not excite the same subpopulation of neurons in all of them.

3. The upper limit of temporal pitch measured at the base of normal-hearing subjects (using harmonic complexes bandpass filtered in a very high frequency region-between 7.8 and $10.8 \mathrm{kHz}$ ) is similar to that found in our CI subjects listening to PSA-Apex pulses (Carlyon and Deeks 2002).

The peripheral explanation is also supported by some evidence suggesting that the electrode-neuron interface is more efficient at the apex of the cochlea. First, behavioural thresholds measured in tripolar configuration were found to be lower for apical stimulation (Bierer 2007) although, in a more recent publication, no such trend was obtained (Bierer and Faulkner 2010). Similarly, van den Honert et al. (2007) found a trend for lower thresholds at the apex using a spatially selective ("phased array") method of stimulation. Second, electrically evoked compound action poten- tial thresholds are usually lower for apical than for basal electrodes (e.g. Botros and Psarros 2010; Brill et al. 2009). Third, Drennan and Pfingst (2005) measured current-level discrimination for bipolar stimuli at two different sites in the cochlea and found better current-level sensitivity for the more apical site. Brill et al. (2009) also reported a steeper increase in ECAP amplitude for stimulation of the most apical electrode of Med-El users. The existence of a better electrodeneuron interface at the apex may relate to two different factors. First, it may reflect a greater density of spiral ganglion cells at the apex, as neural degeneration usually affects the base of the cochlea more than the apex (Zimmermann et al. 1995). Second, it may relate to a closer positioning of the electrode from the modiolus because the cochlea diminishes in size from base to apex. Although there is no direct evidence that pitch discrimination is affected by neural survival or by the distance of the electrode to the neural elements, Moore and Carlyon (2005) re-analysed some data published by Pfingst (1993) and showed that rate DLs at 100 pps correlated with duration of deafness. As neural degeneration is expected to be more pronounced in subjects with a long duration of deafness, we may expect temporal pitch cues to be more effectively conveyed when stimulating high neural density regions. Therefore, although it is possible that a specialized pathway originating at the apex of the cochlea exists in CI users, it is also likely that the improved performance we observed at the apex at least partly reflects the existence of a better electrode-neuron interface.

Implementation in a speech-processing strategy. Several CI strategies have been developed to explicitly code the fundamental frequency (F0) of sounds (Green et al. 2005; Laneau et al. 2006; Milczynski et al. 2009). These strategies usually modulate all channels of the implant in phase. While such techniques have improved the perception of pitch, Green et al. (2005) also showed that they could impair speech perception. An alternative to these strategies may be to explicitly code the F0 only on one channel of the implant. The concept of having one "pitch channel" to convey temporal cues has been investigated by Hamilton et al. (2007). They used monopolar stimulation and tested a modified strategy with F0cues presented either on the most apical or on the most basal channel. The benefits of this strategy on the perception of voice-pitch appeared limited compared to a standard "CIS" strategy and no consistent effect of the pitch channel location was observed, although a few subjects performed better in the basal-channel condition. Our results suggest that a pitch channel may be chosen by selecting the channel with the lowest MCL. Such a strategy may only work in 
the case of focused stimulation as was the case here (e.g. using bipolar or tripolar) because MCLs measured in monopolar mode are usually more homogeneous across channels (Bierer 2007). Alternatively, for devices allowing the use of asymmetric pulses, temporal pitch cues may be consistently conveyed over a wide range of pulse rates by bipolar PSA-Apex pulses.

Limitations on voltage compliance impose the use of fairly long phase durations (here $100 \mu$ s for the short phase) to reach MCL with bipolar pseudomonophasic pulses. However, a CI speech-processing strategy using such pulses only on the most apical channel, together with monopolar stimulation at other sites, could be realistically implemented.

\section{ACKNOWLEDGMENTS}

We thank the subjects for thier participation and Peter Watson for statistical advice. Two anonymous reviewers provided helpful comments on a previous version of this manuscript. This research was supported by the Wellcome trust (grant number 080216).

\section{Open Access}

This article is distributed under the terms of the Creative Commons Attribution Noncommercial License which permits any noncommercial use, distribution, and reproduction in any medium, provided the original author(s) and source are credited.

\section{APPENDIX}

The ranking data obtained with the mid-point comparison procedure were analysed in two different ways presented below.

\section{Broken-stick fit}

For each condition, the upper limit value was obtained by fitting the following function to the data:

$$
\hat{y}=a x+b-a(x-c) H(x-c)
$$

where $a, b$ and $c$ are the unknown and $c$ is the rate value corresponding to the knee point (the so-called upper limit). $H$ is the unit step function:

$$
\begin{cases}H(x)=0 & \text { if } x<0 \\ H(x)=1 & \text { if } x \geq 0\end{cases}
$$

On some occasions, the perceived pitch first increased and then decreased (e.g. S7 in experiment 1). In this case, we allowed the slope of the otherwise constant portion to be negative. For subjects for whom there was a significant difference between the three higher rates of the condition, the upper limit was assumed to be the highest rate tested.

\section{Slope analysis}

Pitch discrimination performance above 300 pps was also assessed by calculating the slopes of increase of the rank functions for rates equal or higher than 344 pps. The ranks were first re-ordered from 1 to 4 (experiment 1 ) or 1 to 5 (experiment 2). This was to avoid slopes higher than 1 which could have been obtained if performance at lower rates was weak. When the slope was negative, it was set to zero in the analysis to avoid overestimating the differences between the conditions that were compared. The following analysis is similar to that described by Carlyon (2000).

The slope of the best-fitting line " $"$ " constrained to pass to $k_{i}$ points obtained in condition $i$ is:

$$
\hat{\beta}_{i}=\frac{\sum_{j=1}^{k} x_{i j} y_{i j}}{\sum_{j=1}^{k} x_{i j}{ }^{2}}
$$

And the summed squared errors in fitting line $i$ is:

$$
S_{i}^{2}=\sum_{j=1}^{k}\left(y_{i j}-\hat{\beta}_{i} x_{i j}\right)^{2}
$$

Comparing two different conditions "1" and "2" (i.e. $i=1$ and $i=2$ ), the mean square error term is:

$$
\hat{\sigma}^{2}=\frac{S_{1}^{2}+S_{2}^{2}}{\left(k_{1}-2\right)+\left(k_{2}-2\right)}
$$

The value of $t$ for the contrast 1 vs. 2 is then:

$$
t=\frac{\hat{\beta}_{1}+\hat{\beta}_{2}}{\sqrt{\hat{\sigma}^{2}\left(x_{1 j}{ }^{2}+x_{2 j}{ }^{2}\right)}}
$$

distributed with $\sum_{i=1}^{2}\left(k_{i}-2\right)$ degrees of freedom.

\section{REFERENCES}

Baumann U, Nobbe A (2004) Pulse rate discrimination with deeply inserted electrode arrays. Hear Res 196:49-57

BIERER JA (2007) Threshold and channel interaction in cochlear implant users: evaluation of the tripolar electrode configuration. J Acoust Soc Am 121:1642-1653

Bierer JA, FAulkner KF (2010) Identifying cochlear implant channels with poor electrode-neuron interface: partial tripolar, 
single-channel thresholds and psychophysical tuning curves. Ear Hear 31:247-258

Bland JM, Altman DG (1995) Calculating correlation coefficients with repeated observations: part 1 -correlation within subjects. BMJ 310:446

Bonham BH, Middlebrooks JC, Rebscher SJ, Snyder RL (2003) The neurophysiological effects of simulated auditory prosthesis stimulation. Third quarterly progress report, NIH contract N01-DC-02-1006

Botros A, Psarros C (2010) Neural response telemetry reconsidered: I. The relevance of ECAP threshold profiles and scaled profiles to cochlear implant fitting. Ear Hear 31:367-379

Brill S, Müller J, Hagen R ET Al (2009) Site of cochlear stimulation and its effect on electrically evoked compound action potentials using the MED-EL standard electrode array. Biomed Eng Online 16:8-40

CARLYON RP (2000) Detecting coherent and incoherent frequency modulation. Hear Res 140:173-188

Carlyon RP, DeEks JM (2002) Limitations on rate discrimination. J Acoust Soc Am 112:1009-1025

Carlyon RP, Deers JM, McKay CM (2010a) The upper limit of temporal pitch for cochlear-implant listeners: stimulus duration, conditioner pulses, and the number of electrodes stimulated. J Acoust Soc Am 127:1469-1478

Carlyon RP, Lynch C, Deeks JM (2010b) Effect of stimulus level and place of stimulation on temporal pitch perception by cochlear implant users. J Acoust Soc Am 127:2997-3008

Carlyon RP, Macherey O, Frijns JHM, Axon PR, Kalkman RK, Boyle P, Baguley DM, Briggs J, Deeks JM, Briaire JJ, Barreau X, Dauman R (2010c) Pitch comparisons between electrical stimulation of a cochlear implant and acoustic stimuli presented to a normal-hearing contralateral ear. J Assoc Res Otolaryngol 11:625-640

Dauman R, Baguley D, Carlyon RP et al (2009) The BordeauxCambridge Programme for unilateral sudden deafness with disabling tinnitus. Assoc Res Otolaryngol Abs 32:154

Drennan WR, PFingst BE (2005) Current-level discrimination using bipolar and monopolar electrode configurations in cochlear implants. Hear Res 202:170-179

FAYAd JN, Linthicum FH JR (2006) Multichannel cochlear implants: relation of histopathology to performance. Laryngoscope 116:1310-1320

Finley CC, Holden TA, Holden LK, Whiting BR, Chole RA, Neely GJ, Hullar TE, SkinNer MW (2008) Role of electrode placement as a contributor to variability in cochlear implant outcomes. Otol Neurotol 29:920-928

Frijns JH, DE SNoo SL, ten Kate JH (1996) Spatial selectivity in a rotationally symmetric model of the electrically stimulated cochlea. Hear Res 95:33-48

Gani M, Valentini G, Sigrist A, Kós Mi, Boex C (2007) Implications of deep electrode insertion on cochlear implant fitting. J Assoc Res Otolaryngol 8:69-83

Green T, Faulkner A, Rosen S, Macherey O (2005) Enhancement of temporal periodicity cues in cochlear implants: effects on prosodic perception and vowel identification. J Acoust Soc Am 118:375-385

GREENWOOD DD (1990) A cochlear frequency-position function for several species-29 years later. J Acoust Soc Am 87:25922605

Hamilton N, Green T, Faulkner A (2007) Use of a single channel dedicated to conveying enhanced temporal periodicity cues in cochlear implants: effects on prosodic perception and vowel identification. Int J Audiol 46:244-253

JEsteAdT W (1980) An adaptive procedure for subjective judgements. Percept Psychophys 28:85-88

KeNDALL DG (1971) Seriation from abundance matrices. In: Hodson FR, Kendall DG, Tautu P (eds) Mathematics in the archeological and historical sciences. Edinburgh University Press, Edinbugh, pp 215-252

Kong YY, CARLYON RP (2010) Temporal pitch perception at high rates in cochlear implants. J Acoust Soc Am 127:3114-3123

Kreft HA, Donaldson GS, Nelson DA (2004) Effects of pulse rate on threshold and dynamic range in Clarion cochlear-implant users. J Acoust Soc Am 115:1885-1888

Krumbholz K, Patterson RD, Pressnitzer D (2000) The lower limit of pitch as determined by rate discrimination. J Acoust Soc Am 108:1170-1180

Laneau J, Boets B, Moonen M, van Wieringen A, Wouters J (2005) A flexible auditory research platform using acoustic or electric stimuli for adults and young children. J Neurosci Meth 142:131136

Laneau J, Wouters J, Moonen M (2006) Improved music perception with explicit pitch coding in cochlear implants. Audiol Neurootol 11:38-52

LevitT H (1971) Transformed up-down methods in psychoacoustics. J Acoust Soc Am 49(Suppl 2):467+

Long CJ, Nimmo-Smith I, Baguley DM, O’Driscoll M, Ramsden R, Otto SR, Axon PR, CarLyon RP (2005) Optimizing the clinical fit of auditory brain stem implants. Ear Hear 26:251-262

MacCallum RC (1977) Effects of conditionality on INDSCAL and ALSCAL weights. Psychometrika 42:297-305

Macherey O, CarLYON RP (2010) Temporal pitch percepts elicited by dual-channel stimulation of a cochlear implant. J Acoust Soc Am 127:339-349

Macherey O, van Wieringen A, Carlyon RP, Deeks JM, Wouters J (2006) Asymmetric pulses in cochlear implants: effects of pulse shape, polarity, and rate. J Assoc Res Otolaryngol 7:253-266

Macherey O, Carlyon RP, van Wieringen A, Deeks JM, Wouters J (2008) Higher sensitivity of human auditory nerve fibers to positive electrical currents. J Assoc Res Otolaryngol 9:241251

Macherey O, van Wieringen A, Cariyon RP, Dhooge I, Wouters J (2010) Forward-masking patterns produced by symmetric and asymmetric pulse shapes in electric hearing. J Acoust Soc Am 127:326-338

Matsuoka AJ, Abbas PJ, Rubinstein JT, Miller CA (2000) The neuronal response to electrical constant-amplitude pulse train stimulation: evoked compound action potential recordings. Hear Res 149:115-128

MCKay CM, Cariyon RP (1999) Dual temporal pitch percepts from acoustic and electric amplitude-modulated pulse trains. J Acoust Soc Am 105:347-357

McKay CM, McDermott H, Carlyon RP (2000) Place and temporal cues in pitch perception: are they truly independent? ARLO $1: 25-30$

McKay CM, Henshall KR, Hull AE (2005) The effect of rate on the perception of spectral shape by cochlear implantees. J Acoust Soc Am 118:386-392

Middlebrooks JC, SNyder RL (2010) Selective electrical stimulation of the auditory nerve activates a pathway specialized for high temporal acuity. J Neurosci 30:1937-1946

Milczynski M, Wouters J, van Wieringen A (2009) Improved fundamental frequency coding in cochlear implant signal processing. J Acoust Soc Am 125:2260-2271

Moon AK, Zwolan TA, Pfingst BE (1993) Effects of phase duration on detection of electrical stimulation of the human cochlea Hear Res 67:166-178

Moore BC, Carlyon RP (2005) Perception of pitch by people with cochlear hearing loss and by cochlear implant users. In Plack CJ, Oxenham AJ, Fay RR, Popper AN (eds) Pitch: neural coding and perception. Springer, New York, pp 234 277 
Oxenham AJ, Bernstein JG, Penagos H (2004) Correct tonotopic representation is necessary for complex pitch perception. Proc Natl Acad Sci USA 101:1421-1425

Pfingst BE (1993) Comparison of spectral and nonspectral frequency difference limens for human and nonhuman primates. J Acoust Soc Am 93:2124-2129

Poulton EC (1979) Models for biases in judging sensory magnitude. Psychol Bull 86:777-803

Rubinstein JT, Wilson BS, Finley CC, Abbas PJ (1999) Pseudospontaneous activity: stochastic independence of auditory nerve fibers with electrical stimulation. Hear Res 127:108-118

SAOJI AA, Litvak LM (2010) Use of "phantom electrode" technique to extend the range of pitches available through a cochlear implant. Ear Hear 31:693-701

Shannon RV (1985) Threshold and loudness functions for pulsatile stimulation of cochlear implants. Hear Res 18:135143

Tong YC, Blamey PJ, Dowell RC, Clark GM (1983) Psychophysical studies evaluating the feasibility of a speech processing strategy for a multiple-channel cochlear implant. J Acoust Soc Am $74: 73-80$
Townshend B, Cotter N, Van Compernolle D, White RL (1987) Pitch perception by cochlear implant subjects. J Acoust Soc Am 82:106-115

Undurraga JA, van Wieringen A, Cariyon RP, Macherey O, Wouters J (2010) Polarity effects on neural responses of the electrically stimulated auditory nerve at different cochlear sites. Hear Res 269:146-161

van den Honert C, Carlyon RP, Long CJ, Smith ZM, Shelton C, Kelsall D (2007) Perceptual evidence of spatially-restricted excitation with focused stimulation. CIAP conference on implantable auditory prostheses, Granlibakken, CA

van Wieringen A, Macherey O, Carlyon RP, Deeks JM, Wouters J (2008) Alternative pulse shapes in electrical hearing. Hear Res 242:154-163

Young FW, TAKane Y, LeWycky R (1978) ALSCAL: a nonmetric multidimensional scaling program with several difference options. Behav Res Meth Instrum 10:451-453

ZENG FG (2002) Temporal pitch in electric hearing. Hear Res 174:101-106

Zimmermann CE, Burgess BJ, Nadol JB Jr (1995) Patterns of degeneration in the human cochlear nerve. Hear Res 90:192-201 